INTERNATIONAL JOURNAL OF RESEARCHES IN BIOSCIENCES, AGRICULTURE AND TECHNOLOGY (C) VISHWASHANTI MULTIPURPOSE SOCIETY (Global Peace Multipurpose Society) R. No. MH-659/13(N) www.vmsindia.org

\title{
ANALYSIS OF STRESS FUNCTIONS IN THIN CIRCULAR DISC
}

\section{S. Warbhe}

Sarvodaya Mahavidyalaya, Sindewahi. Dist. Chandrapur (M.S.) drmwarbhe@gmail.com

\section{Abstract:}

In this paper, an attempt is made to determine the temperature, displacement and stress functions, in thin circular disc of thickness $2 \mathrm{~h}$ occupying the space $\mathrm{D}$ : $\mathrm{a} \leq \mathrm{r} \leq \mathrm{b},-\mathrm{h} \leq \mathrm{z} \leq \mathrm{h}$ by applying finite Marchi-Fasulo integral transform and laplace transform.

Keywords: Circular disc, Marchi-Fasule transform, laplace transform

\section{Introduction:}

Nowacki (1957) has determined unsteady state thermal stresses in circular disc subjected to an exisymmetric temperature distribution on the upper face with zero temperature. On the lower face and the circular edge. Further Roychoudhari (1972) succeeded in determining the quasistatic thermal stresses in a thin circular plate subjected to transient temperature along the circumference of circular palte over the upper face with lower face at zero temperature and fixed circular edge thermally insulated. Wankhede (1982) determined the quasi static thermal stresses in a thin circular plate subjected to arbitrary initial temperature on the upper face with lower face at zero temperature. Ishihara et. al. (1997) considered a circular plate and discussed the transient thermo elasto plastic bending problem making use of the strain increment theorem. In this problem an attempt has been made to solve the transient state problem of thermoelastic deformation of thin circular disc where homogeneous boundry condition of third kind is maintained at $g(z)$. And temperature displacement and stress functions of circular disc of thickness $2 \mathrm{~h}$ occupying the space $\mathrm{D}$ : $\mathrm{a} \leq$ $r \leq b,-h \leq z \leq h h$ is found out. This result is more useful in engineering problem.

\section{STATEMENT OF THE PROBLEM}

Consider a thin circular disc of thickness $2 \mathrm{~h}$ occupying the space $\mathrm{D}$ : $\mathrm{a} \leq \mathrm{r} \leq \mathrm{b}$, $\mathrm{h} \leq \mathrm{z} \leq \mathrm{h}$. The D.E. governing the displacement function $\mathrm{U}(\mathrm{r}, \mathrm{z}, \mathrm{t})$ is given by

$$
\frac{\partial^{2} U}{\partial r^{2}}+\frac{1}{r} \frac{\partial U}{r r}=(1+v) a_{t} \cdot T
$$

With $\mathrm{U}=0$ at $\mathrm{r}=\mathrm{a}$ and $\mathrm{r}=\mathrm{b}$

$r$ and are the Psissions ratio and the linear coefficient of thermal expansion of the af the material of the disc respectively and $T(r, z, t)$ is the temperature of the disc satisfying the D. E.

$$
\frac{\partial^{T}}{r r^{2}}+\frac{1}{r} \frac{\partial T}{\partial r}+\frac{\partial^{2} T}{\partial z^{2}}=\frac{1}{k} \frac{\partial T}{\partial t}
$$

subject to the initial condition

$\mathrm{T}(\mathrm{r}, \mathrm{z}, 0)=0$

And the boundry conditions

$$
\begin{aligned}
& \left\{T(r, z, t)+\frac{\partial}{\partial r} T(r, z, t)\right\}_{r=a)}=g(z, t) \\
& \left\{T(r, z, t)+\frac{\partial}{\partial r} T(r, z, t)\right\}_{(r=b)}=f(x, t) \\
& \left\{T(r, z, t)+k_{1} \frac{\partial}{\partial r} T(r, z, t)\right\}_{(z=-h)}=0 \\
& \left\{T(r, z, t)+k_{2} \frac{\partial}{\partial r} T(r, z, t)\right\}_{z=h)}=0
\end{aligned}
$$

Stress functions are given by

$$
\begin{gathered}
\sigma_{r r}=\frac{-2 \mu}{r} \cdot \frac{\partial U}{\partial r^{2}} \\
\sigma_{\theta \theta}=-2 \mu \frac{\partial^{2} U}{r r^{2}}
\end{gathered}
$$

Where $\mu$ is the lame's constant while each

of the stress functions $\sigma_{r z}, \sigma_{z z}, \sigma_{\theta z}$ are the zero within the disc in the plane state of stress. The equations (1) to (10) construct the mathematical formulation of the problem under consideration.

\section{SOLUTION OF THE PROBLEM}

Applying the finite Marci-Fasulo integral transform to equations (3), (4), (5), (6) and using equations (7), (8) gives

$$
\begin{gathered}
\frac{\partial^{2} \bar{T}}{r r^{2}}+\frac{1}{r} \cdot \frac{\partial \bar{T}}{\partial r}-a_{n}^{2 \cdot} \bar{T}=\frac{1}{k} \cdot \frac{\partial \bar{T}}{\partial t} \\
\bar{T}(r, n, 0)=0 \\
\left\{\bar{T}(r, n, t)+\frac{\partial \bar{T}(r, n, t)}{r r}\right\}=\bar{g}(n, t) \\
\left\{\bar{T}(r, n, t)+\frac{\partial \bar{T}(r, n, t)}{r r}\right\}_{=b}=\bar{f}(n, t)
\end{gathered}
$$

Where denotes the Marchi- Fasulo transform of $\mathrm{T}$ and $\mathrm{n}$ is the Marchi-Fasulo transform parameter $\alpha_{1} \alpha_{2}, \beta_{1}, \beta_{2}$ are constant. 
I J R B A T, Issue (3), Vol. II, May 2015: 419-423

ISS $2347-517 X$

Applying laplace transform and their inverse on equation (3.1) to (3.4), one obtains the temperature $\mathrm{T}(\mathrm{r}, \mathrm{z}, \mathrm{t})$ as

$$
\begin{aligned}
& T(r, z, t)=\sum_{n=1}^{\infty} \frac{P_{n}(z)}{\lambda_{n}} \sum_{m=1}^{\infty} \frac{\lambda_{m^{2}}\left[y_{0}\left(\lambda_{m} a\right)+\lambda_{m} Y_{0}^{\prime}\left(\lambda_{m} a\right)\right]\left[y_{0}\left(\lambda_{m} b\right)+\lambda_{m} Y_{0}^{\prime}\left(\lambda_{m} b\right)\right]}{\left(\lambda_{m}^{2}+a_{n}^{2}\right)\left(\left[y_{0}\left(\lambda_{m} a\right)+\lambda_{m} Y_{0}^{\prime}\left(\lambda_{m} a\right)\right]^{2}-\left[y_{0}\left(\lambda_{m} b\right)+\lambda_{m} Y_{0}^{\prime}\left(\lambda_{m} b\right)\right]^{2}\right)} \\
& \mathrm{X}^{\lambda_{m}\left[Y_{0}\left(\lambda_{m} a\right)+\lambda_{m} Y_{0}^{\prime}\left(\lambda_{m} a\right)\right]\left[J_{0}\left(\lambda_{m} r\right)+\lambda_{m} J_{0}^{\prime}\left(\lambda_{m} r\right)\right]} \\
& { }_{-}\left[Y_{0}\left(\lambda_{m} r\right)+\lambda_{m} Y_{0}^{\prime}\left(\lambda_{m} r\right)\right]\left[J_{0}\left(\lambda_{m} a\right)+\lambda_{m} J_{0}^{\prime}\left(\lambda_{m} a\right)\right] \\
& \int^{t} f\left(n, t^{\prime}\right) \cdot e^{-k\left(\lambda_{m}^{2}+a_{n}^{2}\right)\left(t-t^{\prime}\right)} \cdot d t \\
& \sum_{n=1}^{\infty} \frac{P_{n}(z)}{\lambda_{n}} \sum_{m=1}^{\infty} \frac{\lambda_{m}^{2}\left[Y_{0}\left(\lambda_{m} b\right)+\lambda_{m} Y_{0}^{\prime}\left(\lambda_{m} b\right)\right]^{2}}{\left(\lambda_{m}^{2}+a_{n}^{2}\right)\left(\left[Y_{0}\left(\lambda_{m} a\right)+\lambda_{m} Y_{0}^{\prime}\left(\lambda_{m} a\right)\right]^{2}-\left[Y_{0}\left(\lambda_{m} b\right)-\lambda_{m} Y_{0}\left(\lambda_{m} b\right)\right]^{2}\right)} \\
& \mathrm{X}^{\lambda_{m}\left[Y_{0}\left(\lambda_{m} b\right)+\lambda_{m} Y_{0}^{\prime}\left(\lambda_{m} b\right)\right]\left[J_{0}\left(\lambda_{m} n\right)+\lambda_{m} J_{0}^{\prime}\left(\lambda_{m} r\right)\right]} \\
& -\lambda_{m}\left[Y_{0}^{\prime}\left(\lambda_{m} r\right)+\lambda_{m} Y_{0}^{\prime}\left(\lambda_{m} r\right)\right] \cdot\left[J_{0}\left(\lambda_{m} b\right)+\lambda_{m} J_{0}^{\prime}\left(\lambda_{m} b\right)\right] \\
& \int_{0}^{t} g\left(n, t^{\prime}\right) \cdot e^{-k\left(\lambda_{m}^{2}+a_{n}^{2}\right)\left(t-t^{\prime}\right)} \cdot d t^{\prime}
\end{aligned}
$$

DETERMINATION OF THERMOELASTIC DISPLACEMENT:

Substituting the values of $\mathrm{T}(\mathrm{r}, z, \mathrm{t})$ from equation (3.5) in equation (3.1) one obtains

$$
\begin{aligned}
& U(r, z, t)=-(1+v) a \sum_{n=1}^{\infty} \frac{P_{n}(z)}{\lambda_{n}} \sum_{m=1}^{\infty} \frac{\left[Y_{0}\left(\lambda_{m} a\right)+\lambda_{m} Y_{0}^{\prime}\left(\lambda_{m} a\right)\right]\left[Y_{0}\left(\lambda_{m} b\right)+\lambda_{m} Y_{0}^{\prime}\left(\lambda_{m} b\right)\right]}{\left(\lambda_{m}^{2}+a_{n}^{2}\right)\left(\left[Y_{0}\left(\lambda_{m} a\right)+\lambda_{m} Y_{0}^{\prime}\left(\lambda_{m} a\right)\right]^{2}-\left[Y_{0}\left(\lambda_{m} b\right)-\lambda_{m} Y_{0}^{\prime}\left(\lambda_{m} b\right)\right]^{2}\right)} \\
& \mathrm{X}\left[Y_{0}\left(\lambda_{m} a\right)+\lambda_{m} Y_{0}^{\prime}\left(\lambda_{m} a\right)\right]\left[J_{0}\left(\lambda_{m} r\right)+\lambda_{m} J_{0}^{\prime}\left(\lambda_{m} r\right)\right] \\
& {\left[Y_{0}\left(\lambda_{m} r\right)+\lambda_{m} Y_{0}^{\prime}\left(\lambda_{m} r\right)\right]\left[J_{0}\left(\lambda_{m} a\right)+\lambda_{m} J_{0}^{\prime}\left(\lambda_{m} a\right)\right]} \\
& \int^{t} f\left(n, t^{\prime}\right) \cdot e^{-k\left(\lambda_{m}^{2}+a_{n}^{2}\right)\left(t-t^{\prime}\right)} \cdot d t^{\prime} \\
& \text {. }\left[Y_{0}\left(\lambda_{m} r\right)+\lambda_{m} Y_{0}^{\prime}\left(\lambda_{m} r\right)\right]\left[J_{0}\left(\lambda_{m} b\right)+\lambda_{m} J_{0}^{\prime}\left(\lambda_{m} b\right)\right] \\
& \int_{0}^{t} g\left(n, t^{\prime}\right) \cdot e^{-k\left(\lambda_{m}^{2}+a_{n}^{2}\right)\left(t-t^{\prime}\right)} \cdot d t^{\prime}
\end{aligned}
$$

420 
I J R B A T, Issue (3), Vol. II, May 2015: 419-423

ISS $2347-517 X$

DETERMINATION OF STRESS FUNCTION

Using (3.6) in (2.9), (2.10) one obtains the expression of stress functions

$$
\begin{aligned}
& \sigma_{r r}=\frac{2 \mu}{r}(1+v) a_{t} \sum_{n=1}^{\infty} \frac{P_{n}(z)}{\lambda_{n}} \sum_{m=1}^{\infty} \frac{\left[Y_{0}\left(\lambda_{m} a\right)+\lambda_{m} Y_{0}^{\prime}\left(\lambda_{m} a\right)\right]\left[Y_{0}\left(\lambda_{m} b\right)+\lambda_{m} Y_{0}^{\prime}\left(\lambda_{m} b\right)\right]}{\left(\lambda_{m}^{2}+a_{n}^{2}\right)\left(\left[Y_{0}\left(\lambda_{m} a\right)+\lambda_{m} Y_{0}^{\prime}\left(\lambda_{m} a\right)\right]^{2}\left[Y_{0}\left(\lambda_{m} b\right)+\lambda_{m} Y_{0}^{\prime}\left(\lambda_{m} b\right)\right]^{2}\right)} \\
& \mathrm{X}^{\lambda_{m}}\left[Y_{0}\left(\lambda_{m} a\right)+\lambda_{m} Y_{0}^{\prime}\left(\lambda_{m} a\right)\right]\left[J_{0}^{\prime}\left(\lambda_{m} r\right)+\lambda_{m} J_{0}^{\prime \prime}\left(\lambda_{m} r\right)\right] \\
& -\lambda_{m}\left[Y_{0}^{\prime}\left(\lambda_{m} r\right)+\lambda_{m} Y_{0}^{\prime}\left(\lambda_{m} r\right)\right]\left[J_{0}\left(\lambda_{m} a\right)+\lambda_{m} J_{0}^{\prime}\left(\lambda_{m} a\right)\right] \\
& x \int_{0}^{t} f\left(n, t^{\prime}\right) \cdot e^{-k\left(\lambda_{m}^{2}+a_{n}^{2}\right)\left(t-t^{\prime}\right)} \cdot d t^{\prime} \\
& \frac{2 \mu}{r}(1+v) a_{t} \sum_{n=1}^{\infty} \frac{P_{n}(z)}{\lambda_{n}} \sum_{m=1}^{\infty} \frac{\left[Y_{0}\left(\lambda_{m} b\right)+\lambda_{m} Y_{0}^{\prime}\left(\lambda_{m} b\right)\right]^{2}}{\left(\lambda_{m}^{2}+a_{n}^{2}\right)\left(\left[Y_{0}\left(\lambda_{m} a\right)+\lambda_{m} Y_{0}^{\prime}\left(\lambda_{m} a\right)\right]^{2}-\left[Y_{0}\left(\lambda_{m} b\right)+\lambda_{m} Y_{0}^{\prime}\left(\lambda_{m} b\right)\right]^{2}\right)}
\end{aligned}
$$

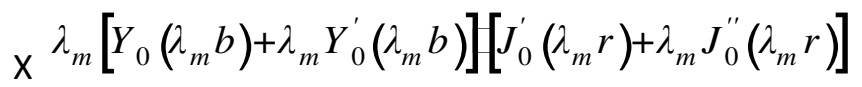

$$
\begin{aligned}
& -\lambda_{m}\left[Y_{0}^{\prime}\left(\lambda_{m} r\right)+\lambda_{m} Y_{0}^{\prime \prime}\left(\lambda_{m} r\right)\right]\left[J_{0}\left(\lambda_{m} b\right)+\lambda_{m} J_{0}^{\prime}\left(\lambda_{m} b\right)\right] \\
& \int_{x}^{t} g\left(n, t^{\prime}\right) \cdot e^{\left.-k\left(\lambda_{m}^{2}+a_{n}^{2}\right) t-t^{\prime}\right)} \cdot d t^{\prime} \\
& \sigma_{\theta \theta}=2 \mu(1+v) a_{t} \sum_{n=1}^{\infty} \frac{P_{n}(z)}{\lambda_{n}} \sum_{m=1}^{\infty} \frac{\left[Y_{0}\left(\lambda_{m} a\right)+\lambda_{m} Y_{0}^{\prime}\left(\lambda_{m} a\right)\right]\left[Y_{0}\left(\lambda_{m} b\right)+\lambda_{m} Y_{0}^{\prime}\left(\lambda_{m} b\right)\right]}{\left(\lambda_{m}^{2}+a_{n}^{2}\right)\left(\left[Y_{0}\left(\lambda_{m} a\right)+\lambda_{m} Y_{0}^{\prime}\left(\lambda_{m} a\right)\right]^{2}-\left[Y_{0}\left(\lambda_{m} b\right)+\lambda_{m} Y_{0}^{\prime}\left(\lambda_{m} b\right)\right]^{2}\right)} \\
& \mathrm{X} \lambda_{m}^{2}\left[Y_{0}\left(\lambda_{m} a\right)+\lambda_{m} Y_{0}^{\prime}\left(\lambda_{m} a\right)\right]\left[J_{0}^{\prime \prime}\left(\lambda_{m} r\right)+\lambda_{m} J_{0}^{\prime \prime \prime}\left(\lambda_{m} r\right)\right] \\
& -\lambda_{m}^{2}\left[Y_{0}^{\prime \prime}\left(\lambda_{m} r\right)+\lambda_{m} Y_{0}^{\prime \prime \prime}\left(\lambda_{m} r\right)\right]\left[J_{0}\left(\lambda_{m} a\right)+\lambda_{m} J_{0}^{\prime}\left(\lambda_{m} a\right)\right] \\
& \mathrm{X} \int_{0}^{t} f\left(n, t^{\prime}\right) \cdot e^{-k\left(\lambda_{m}^{2}+a_{n}^{2}\right)\left(t-t^{\prime}\right)} \cdot d t^{\prime} \\
& \frac{2 \mu}{r}(1+v) a_{t} \sum_{n=1}^{\infty} \frac{P_{n}(z)}{\lambda_{n}} \sum_{m=1}^{\infty} \frac{\left[Y_{0}\left(\lambda_{m} b\right)+\lambda_{m} Y_{0}^{\prime}\left(\lambda_{m} b\right)\right]^{2}}{\left(\lambda_{m}^{2}+a_{n}^{2}\right)\left(\left[Y_{0}\left(\lambda_{m} a\right)+\lambda_{m} Y_{0}^{\prime}\left(\lambda_{m} a\right)\right]^{2}-\left[Y_{0}\left(\lambda_{m} b\right)+\lambda_{m} Y_{0}^{\prime}\left(\lambda_{m} b\right)\right]^{2}\right)} \\
& \mathrm{X} \lambda_{m}^{2}\left[Y_{0}\left(\lambda_{m} b\right)+\lambda_{m} Y_{0}^{\prime}\left(\lambda_{m} b\right)\right]\left[J_{0}^{\prime \prime}\left(\lambda_{m} r\right)+\lambda_{m} J_{0}^{\prime \prime \prime}\left(\lambda_{m} r\right)\right] \\
& -\lambda_{m}^{2}\left[Y_{0}^{\prime \prime}\left(\lambda_{m} r\right)+\lambda_{m} Y_{0}^{\prime \prime \prime}\left(\lambda_{m} r\right)\right]\left[J_{0}\left(\lambda_{m} b\right)+\lambda_{m} J_{0}^{\prime}\left(\lambda_{m} b\right)\right] \\
& x \int_{0}^{t} g\left(n, t^{\prime}\right) \cdot e^{-k\left(l_{m}^{2}+a_{n}^{2}\right)\left(t-t^{\prime}\right)} \cdot d t^{\prime}
\end{aligned}
$$

421 


\section{SPECIAL CASE}

Set $g(z, t)=(z-h)^{2}(z+h)^{2}\left(1-e^{-t}\right)(a+1)$

$f(z, t)=(z-h)^{2}(z+h)^{2}\left(1-e^{-t}\right)(b+1)$

Applying finite Marchi-Fasulo integral transform to the equation (6.1) and (6.2), one obtains

$$
\begin{aligned}
& \bar{f}(z, t)=\int_{-h}^{h}(z-h)^{2}(z+h)^{2}\left(1-e^{-t}\right)(b+1) P_{n}(z) \cdot d z \\
& =4\left(k_{1}+k_{2}\right)\left(1-e^{-t}\right)(b+1)\left[\frac{\left.\left(a_{n} h\right) \cos ^{2}\left(a_{n} h\right)-\cos \left(a_{n} h\right) \sin \left(a_{n} h\right)\right]}{a_{n^{2}}}\right] \\
& \bar{g}(z, t)=\int_{-h}^{h}(z-h)^{2}(z+h)^{2}\left(1-e^{-t}\right)(a+1) P_{n}(z) \cdot d z \\
& =4\left(k_{1}+k_{2}\right)\left(1-e^{-t}\right)(a+1)\left[\frac{\left(a_{n} h\right) \cos ^{2}\left(a_{n} h\right)-\cos \left(a_{n} h\right) \sin \left(a_{n} h\right)}{a_{n^{2}}}\right]
\end{aligned}
$$

Substituting the values of from (5.3) and from (5.4) in equation (3.5)

$$
\begin{aligned}
& T(r, z, t)=4\left(k_{1}+k_{2}\right)(b+1) \sum_{n=1}^{\infty} \frac{P_{n}(z)}{\lambda_{n}} \sum_{m=1}^{\infty} \frac{\lambda_{m^{2}}\left[y_{0}\left(\lambda_{m} a\right)+\lambda_{m} Y_{0}^{\prime}\left(\lambda_{m} a\right)\right]\left[y_{0}\left(\lambda_{m} b\right)+\lambda_{m} Y_{0}^{\prime}\left(\lambda_{m} b\right)\right]}{\left(\lambda_{m}^{2}+a_{n}^{2}\right)\left[y_{0}\left(\lambda_{m} a\right)+\lambda_{m} Y_{0}^{\prime}\left(\lambda_{m} a\right)\right]^{2}-\left[y_{0}\left(\lambda_{m} b\right)+\lambda_{m} Y_{0}^{\prime}\left(\lambda_{m} b\right)\right]^{2}} \\
& \mathrm{x}\left[Y_{0}\left(\lambda_{m} a\right)+\lambda_{m} Y_{0}^{\prime}\left(\lambda_{m} a\right)\right]\left[J_{0}\left(\lambda_{m} r\right)+\lambda_{m} J_{0}^{\prime}\left(\lambda_{m} r\right)\right] \\
& \text { - }\left[Y_{0}\left(\lambda_{m} r\right)+\lambda_{m} Y_{0}^{\prime}\left(\lambda_{m} r\right)\right]\left[J_{0}\left(\lambda_{m} a\right)+\lambda_{m} J_{0}^{\prime}\left(\lambda_{m} a\right)\right] \\
& x \int_{0}^{t}\left(1-e^{-t}\right)\left[\frac{\left(a_{n} h\right) \cos ^{2}\left(a_{n} h\right)-\cos \left(a_{n} h\right) \cdot \sin \left(a_{n} h\right)}{a_{n}^{2}}\right] e^{-k\left(l_{m}^{2}+a_{n}^{2}\right)^{(-i)}} \cdot d t \\
& 4\left(k_{1}+k_{2}\right)(a+1) \sum_{n=1}^{\infty} \frac{P_{n}(z)}{\lambda_{n}} \sum_{m=1}^{\infty} \frac{\lambda_{m}^{2}\left[y_{0}\left(\lambda_{m} b\right)+Y_{o}^{\prime}\left(\lambda_{m} b\right)\right]^{2}}{\left(\lambda_{m}^{2}+a_{n}^{2}\right)\left[y_{0}\left(\lambda_{m} a\right)+\lambda_{m} Y_{0}^{\prime}\left(\lambda_{m} a\right)\right]^{2}-\left[y_{0}\left(\lambda_{m} b\right)+\lambda_{m} Y_{0}^{\prime}\left(\lambda_{m} b\right)\right]^{2}} \\
& x\left[Y_{0}\left(\lambda_{m} b\right)+\lambda_{m} Y_{0}^{\prime}\left(\lambda_{m} b\right)\right]\left[J_{0}\left(\lambda_{m} r\right)+\lambda_{m} J_{0}^{\prime}\left(\lambda_{m} r\right)\right] \\
& -\left[Y_{0}\left(\lambda_{m} r\right)+\lambda_{m} Y_{0}^{\prime}\left(\lambda_{m} r\right)\right]\left[J_{0}\left(\lambda_{m} b\right)+\lambda_{m} J_{0}^{\prime}\left(\lambda_{m} b\right)\right] \\
& x \int_{0}^{t}\left(1-e^{-t}\right)\left[\frac{\left(a_{n} h\right) \cos ^{2}\left(a_{n} h\right)-\cos \left(a_{n} h\right) \cdot \sin \left(a_{n} h\right)}{a_{n}^{2}}\right] e^{-k\left(l_{m}^{2}+a_{n}^{2}\right)^{(-i)}} \cdot d t
\end{aligned}
$$

\section{Conclusion:}

In this problem, I discussed completely yhe transient state problem of thermoelastic deformation of thin circular disc where the homogenous boundry condition of third kind is maintained on the plane surface of disc on the inner curved surface, the third kind boundry condition is maintained at $g(z)$ which is known function of $Z$. 
The finite Marchi-Fasulo integral transform and lapalce transform technique is used to obtain the numerical result. The temperature, displacement and thermal stresses that are obtained can be applied to the design of useful structure or machines in engineering application.

\section{Reference:}

[1] Deshmukh K. C. And Meshram S. A.: "Inverse thermoelastic problem in a thin circular plate", Bull. Of Pure and Appl. Sci. Vol. 20E (No.1), (2001), pp 113-122.

[2] Grysa K and Cialkowski M. J.: "On a certain inverse problem of temperature and thermal stress fields", Acta Mechanica 36 (1980), pp. 169-185.

[3] Grsya $\mathbf{K}$ and Kozlowski Z.: "One dimensional problem of temperature and heat flux determination at the surfaces of a thermoelastic slb part-I, The Analytical Solution”, NUCL. Engrg 74 (1982), pp. 1-14.
[4] Nowacki W.: The state of stress in a thick circular plate due to temperature fieldâ€ , Bull. Sci. Acad. Polon Ser. Tech. 5, (1957), p 227.

[5] Patel S. R.: "Inverse problems of transient heat conduction with radiation", the Mathematics Education, Vol. 5, No. 4, (1971), pp. 85-90.

[6] Roy Chaudhary S. K.: "A note on the quasistatic stresses in a thin circular plate due to transient temperature applied along the circumference of a circle over the uppar face", Bull. Acad. Sci. Ser. Tech. 20 (1972), pp. 21-24.

[7] Wankhede P. C.: "On the quasi-static thermal stresses in a circular plate", Indian J. Of Pure and Appl. Maths 13, (1982), pp. 12731277. 